\title{
Contribution of Different Land Evaluation Systems to Assess Land Capability and Suitability of Some Coastal Soils in Egypt
}

\author{
Ibraheem A.H. Yousif ${ }^{1}$, Sayed A. Hassanein ${ }^{1}$, Ali A. Abdel Hady', Abdalsamad A.A. Aldabaa²
}

10.18805/IJARe.A-497

\begin{abstract}
The objectives of this study were to assess the land capability using Storie Index and Cervatana model and to assess the land suitability by LUSET and Almagra model for some coastal soils in Egypt. Twenty-seven soil profiles were dug and morphologically described to represent all physiographic units. Landsat image, DEM, geological map, field and laboratory work were used to create physiographic-soil map relationship. Based on the modified Storie Index, soils were classified into four land capability grades (grade 2, 3, 4 and 5). The Cervatana model classified these soils into three capability classes, S2, S3 and N. Almagra model indicated that $4.71 \%$ of the area is highly suitable (S2) for wheat and citrus and $14.82 \%$ of the area is S2 for olives. About $31.78 \%$ of the soils is moderately suitable (S3) for wheat and citrus where-as $52 \%$ are S3 for olives. Based on LUST, about $5.85,3.73$ and $2.11 \%$ of soils are highly suitable (S1) for wheat, cotton and olives respectively. About $31 \%$ of the area is moderately suitable (S2) for citrus and peach where-as $63.86 \%$ is S2 for alfalfa and $85 \%$ of the area is S2 for wheat. Soil salinity, calcium carbonate, drainage and soil texture were the most common limiting factors of the soils. The study revealed that the MicroLEIS application either Cervatana or Almagra is not suited to predict the land suitability and land capability while the LUSET and Modified Storie index is recommended for Egyptian pedoenvironment.
\end{abstract}

Key words: Almagra, Cervatana, Land evaluation, LUSET, Storie index.

\section{INTRODUCTION}

Land capability and suitability assessment become a necessary process for defining the potential capabilities of the land under different uses for sustainable land management. Scientifically each specific land unit should be utilized for an application which is suitable for that application (FAO, 1976). Agricultural land suitability is a very significant piece of information in agriculture development and planning (Chiranjit and Kishore, 2018; Ramamurthy et al., 2019). Therefore, there is an intense need for land evaluation studies to select the superior land use (Zhang et al., 2015; Sabareeshwari et al., 2018). Combination of geographic information system with soil survey and land methods were developed and adopted to evaluate soil suitability for different crops (Bhaskar, et al., 2015). Many systems have been designed and developed for land evaluation assessment such as Storie Index (Storie, 1973), land capability classification system (Klingebiel and Montgomery, 1961), FAO Framework for Land Evaluation (FAO 1976), Soil Productivity Index (Delgado 2003), Land Use Suitability Evaluation Tool (LUSET) (Yen et al., 2006), Modified Storie Index (UCDAVIS, 2008), Microcomputer Land Evaluation Information System (MicroLEIS) (De La Rosa et al., 2009) and the Agriculture Land Evaluation System (ALESarid and modified ALESarid-GIS) (Ismail et al., 2005). Land evaluation systems could be either qualitative or quantitative methods. The qualitative approach gives the results in qualitative terms where the quantitative approach involves more parametric techniques which allow various statistical analyses to be performed. This study focused on the comparison of modified Storie Index (UCDAVIS, 2008) and Cervatana
'Soil Science Department, Faculty of Agriculture, Cairo University, Giza, 12613, Egypt.

2Pedology Department, Water Resources and Desert Soils Division, Desert Research Center, Egypt.

Corresponding Author: Ibraheem A.H. Yousif, Soil Science Department, Faculty of Agriculture, Cairo University, Giza, 12613, Egypt. Email: ibraheemyousif@agr.cu.edu.eg

How to cite this article: Yousif, I.A.H., Hassanein, S.A., Hady, A.A.A. and Aldabaa, A.A.A. (2020). Contribution of Different Land Evaluation Systems to Assess Land Capability and Suitability of Some Coastal Soils in Egypt. Indian Journal of Agricultural Research. 54(3): 263-276.

Submitted: 19-10-2019 Accepted: 20-12-2019 Published: 18-03-2020

model of MicroLEIS (De la Rosa et al., 2004) and also comparison between (LUSET) (Yen et al., 2006) and of Almagra model of MicroLEIS (De la Rosa et al., 2004) as land suitability evaluation systems. Many researchers used these systems or built up their own systems depend on the methodology of the soil science (Xingwu et al., 2015). Modified Storie Index was used in several studies to evaluate the land capability in the northwestern coast of Egypt and in many other areas (Sawy, et al., 2013; Abd El-Aziz, 2018; Yousif, 2018; Yousif and Ahmed, 2019). Cervatana and Almagra models of MicroLEIS were applied to assess the land capability and suitability evaluation in many areas in Mediterranean region (Abd El-Aziz, 2018; Abd-Elmabod et al. 2019; Mahmoud et al. 2019; Yousif, 2019). LUSET is a utility tool of land suitability Evaluation for multiple crops. It is programmed in Microsoft Excel and the calculation in 
LUSET was coded using Visual basic for Application (VBA), (Yen et al., 2006) and depends on the land evaluation framework of the FOA (FAO, 1976). LUSET tool was used to assess land suitability evaluation in many different areas (Aldabaa, 2018; Yousif, 2018). The objectives of the current study were to (1) characterize the soils of the area extended from El-Kasaba village to Paghoush village and located at the east of Matrouh city, Egypt. (2) Assess land capability using modified Storie Index and Cervatana model of MicroLEIS. (3) Assess land suitability by LUSET and Almagra model of MicroLEIS.

\section{MATERIALS AND METHODS Study area}

The study area is located in the east of Matrouh city by about $25 \mathrm{~km}$ and it is extended from El-Kasaba village to Paghoush village. It occupies an area of $197.22 \mathrm{~km}^{2}$ (46957.14 Fadden) and located between longitudes $27^{\circ} 25^{\prime} 24^{\prime \prime}$ to $27^{\circ} 47^{\prime} 50^{\prime \prime} \mathrm{E}$ and latitudes $31^{\circ} 7^{\prime} 26^{\prime \prime}$ to $31^{\circ} 13^{\prime} 41^{\prime \prime} \mathrm{N}$ (Fig 1). The elevation ranged between 11-120 m ASL. Flat to nearly level, gently sloping and sloping are the dominant slope classes (Fig 2). Normalized Difference Vegetation Index (NDVI) showed that some areas covered by scattered vegetation with maximum value of 0.5 (Fig 3). The investigated area is characterized by dry hot summer where the mean monthly temperature ranged between 14.5 to $26.7^{\circ} \mathrm{C}$ and almost rainy winter where the annual rainfall ranged between 87.10 and $274.50 \mathrm{~mm}$ year $^{-1}$ with an average of $145.06 \mathrm{~mm}_{\text {year }}{ }^{-1}$ (E.M.A., 2014). It is dominated by a sedimentary rock varying from Tertiary (Miocene) to Quaternary period (El Shazly et al., 1975).

\section{GIS and remote sensing}

Using ArcGIS 10.5, Landsat 8 OLI image (path 179, row 38) captured in 2018 and 3D presentation created from digital elevation model (DEM) were used to distinguish and delineate the different physiographic units.

\section{Field work and laboratory analysis}

To represent all physiographic units, twenty-seven soil profiles were dug and morphologically described according to FAO (2006). Soil analyses were done according to USDA (2017). Soils were classified according to Soil Survey Staff (2014).

\section{Land Evaluation Methods}

\section{Land capability classification}

1. MicroLEIS Cervatana model, De la Roza, 2000 (Table 1). 2. Modified Storie Index Rating, UCDVVIS, 2008 (Table 2). Land suitability classification

1. MicroLEIS, De la Roza (2000), Almagra model (Table 3). 2. Land Use Suitability Evaluation Tool (LUSET), Yen et al., 2006 (Table 4).

\section{RESULTS AND DISCUSSION}

Each landform was represented by some soil profiles as shown in Fig 4 and Table 5 and soils were characterized as the following:

\section{Soils of upper slope unit}

This unit is located in the southern part of the studied area and occupies an area of $51.77 \mathrm{~km}^{2}$ and represented by eight soil profiles (Table 5). Results reveled that most of soils are

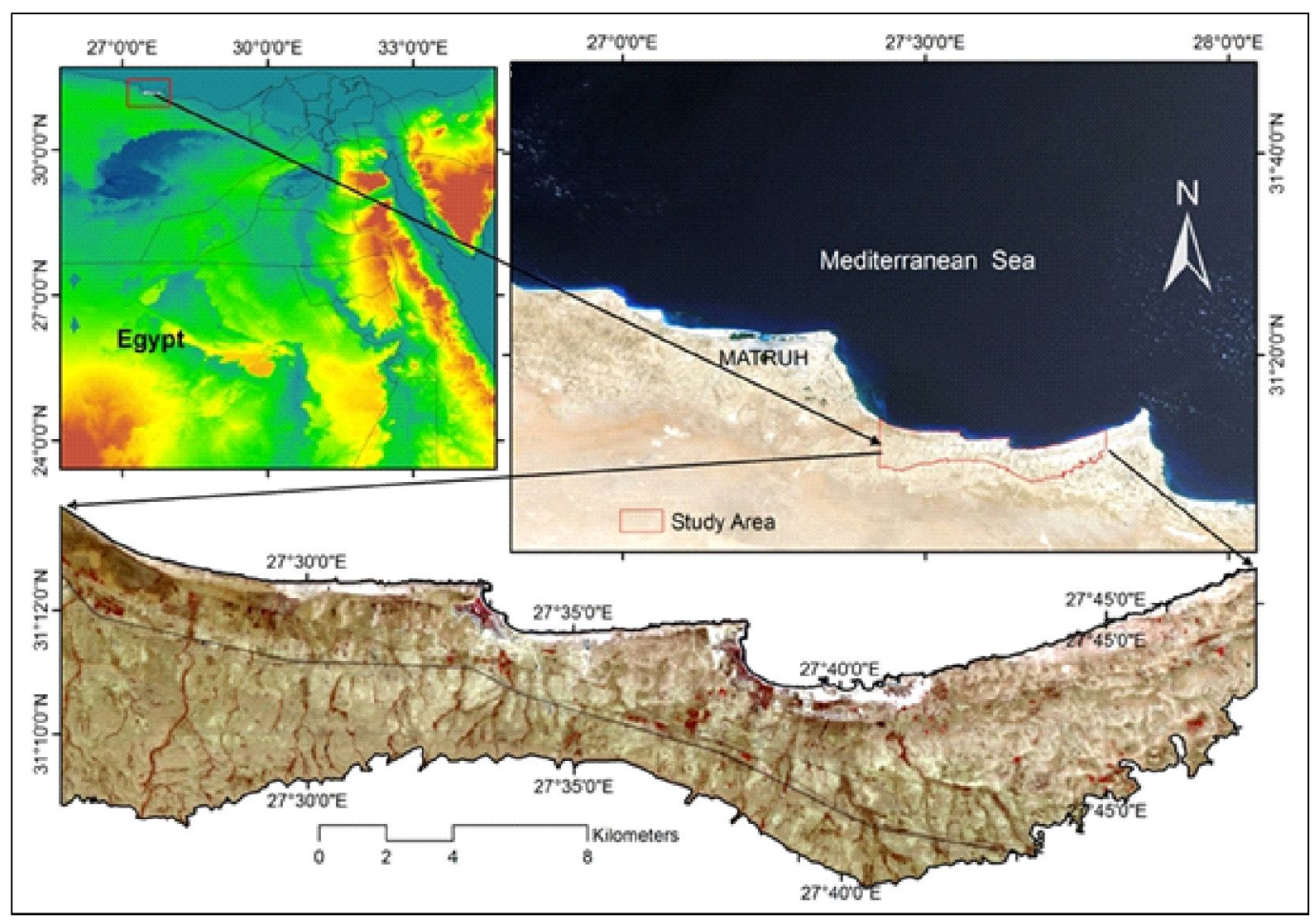

Fig 1: Location map of the investigated area. 


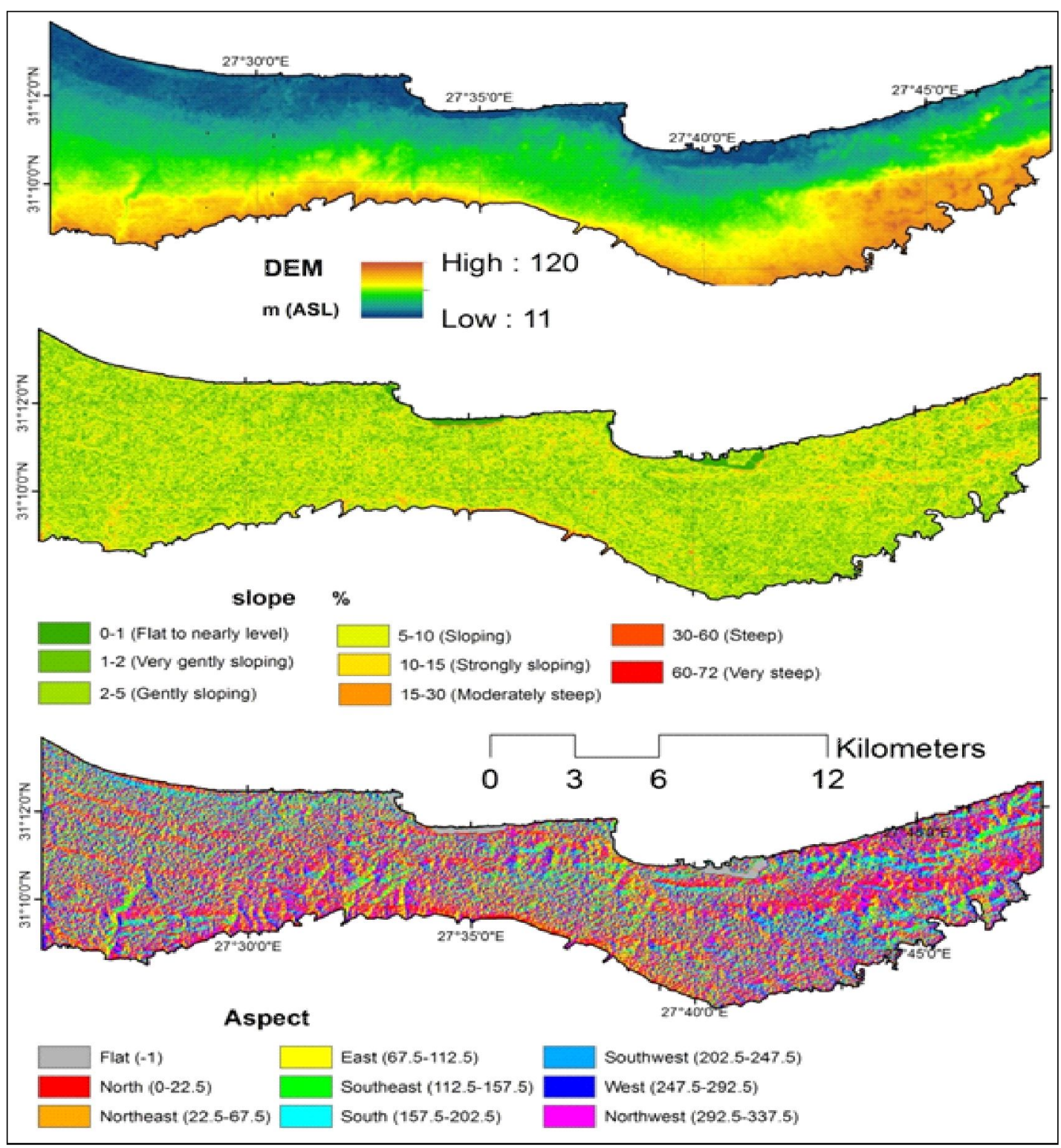

Fig 2: Topographical analysis of the investigated area.

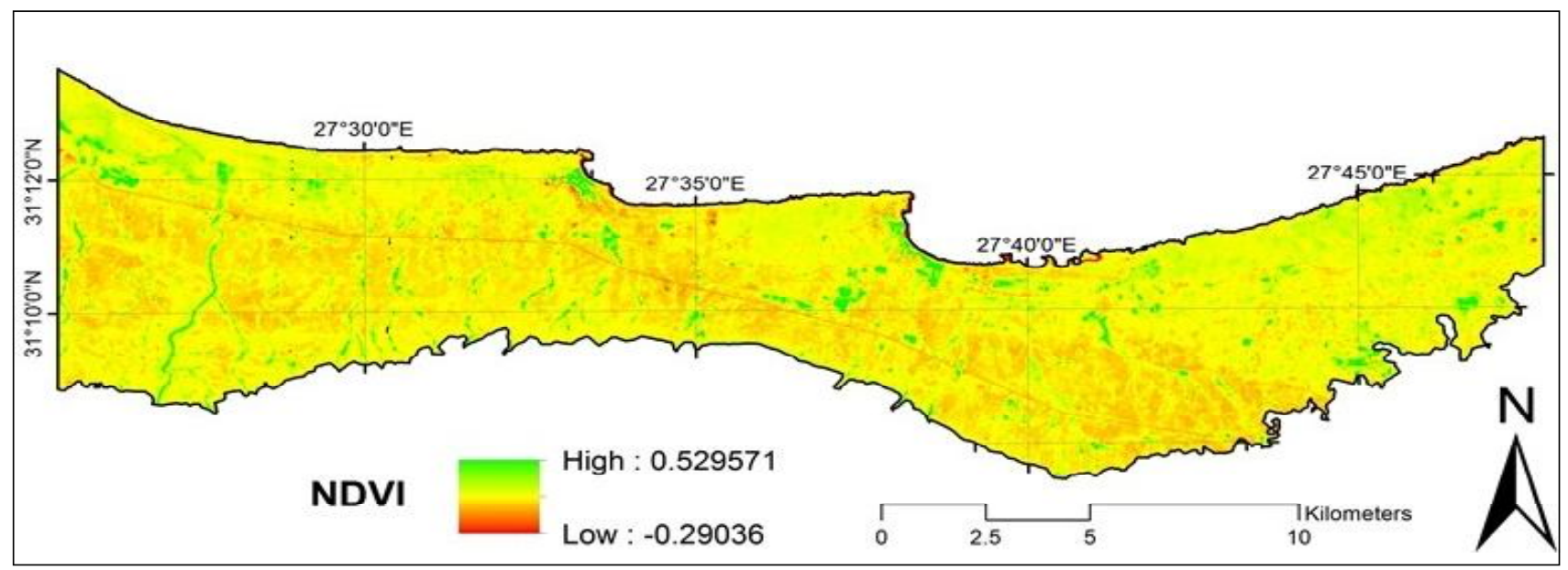

Fig 3: Normalized Difference Vegetation Index (NDVI). 
Contribution of Different Land Evaluation Systems to Assess Land Capability and Suitability of Some Coastal Soils in Egypt

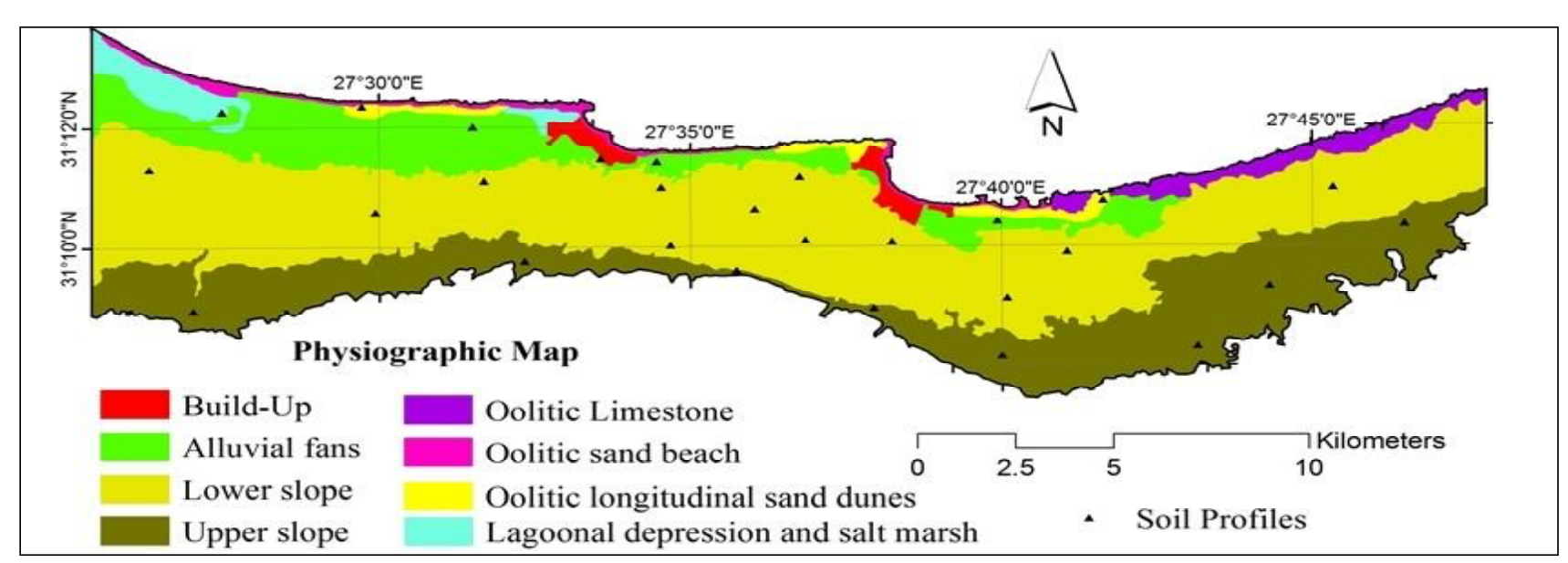

Fig 4: Physiographic soil map.

Table 1: Land capability according to Cervatana model De la Roza (2000).

\begin{tabular}{|c|c|c|c|c|}
\hline Class & Description & Sub class & \multicolumn{2}{|c|}{ Limitation } \\
\hline S1 & Excellent use capability & Slope & $t$ & Slope \\
\hline S2 & Good use capability. & Soil & I & Useful depth \\
\hline S3 & Moderate use capability. & & & Texture class \\
\hline \multirow[t]{3}{*}{ N } & Marginal or non-productive & & & $\begin{array}{l}\text { Stoniness and rockiness } \\
\text { Drainage class }\end{array}$ \\
\hline & & Erosion risk & r & $\begin{array}{l}\text { Soil Erodibility } \\
\text { Slope gradient } \\
\text { Vegetation density }\end{array}$ \\
\hline & & Bioclimatic deficiency & $b$ & $\begin{array}{l}\text { Aridity degree } \\
\text { Frost risks }\end{array}$ \\
\hline
\end{tabular}

Table 2: Land capability classification according to revised Storie Index (2008).

\begin{tabular}{llll}
\hline Class & Description & Rate $\%$ & Limitation severity \\
\hline 1 & Excellent & $80-100$ & No limitation \\
2 & Good & $60-80$ & Slight limitation \\
3 & Fair & $40-60$ & Moderate limitation \\
4 & Poor & $10-40$ & Sever limitation \\
5 & Non-agricultural & $0-10$ & Very sever limitation \\
\hline
\end{tabular}

Table 3: Land suitability according to Almagra model De la Roza (2000).

\begin{tabular}{lllll}
\hline Class & Description & Rate $\%$ & Sub class & Limitation \\
\hline S1 & highly suitable & $100-80$ & $\mathrm{p}$ & Depth \\
S2 & Suitable & $80-60$ & $\mathrm{t}$ & Texture \\
S3 & moderately suitable & $60-40$ & $\mathrm{~d}$ & Drainage \\
S4 & marginal suitable & $40-20$ & $\mathrm{c}$ & Carbonates calcium content \\
S5 & not suitable & $0-20$ & $\mathrm{~s}$ & Salinity \\
& & $\mathrm{a}$ & Sodium saturation \\
& & $\mathrm{g}$ & Development of soil profile \\
\hline
\end{tabular}

Table 4: Land suitability classes according to Yen et al. (2006).

\begin{tabular}{llll}
\hline Class & Description & Rate \% & Limitation severity \\
\hline S1 & Highly suitable & $85-100$ & No limitation \\
S2 & moderately suitable & $60-85$ & Slight limitation \\
S3 & marginally suitable & $40-60$ & Moderate limitation \\
N & not suitable & $0-40$ & Very sever limitation \\
\hline
\end{tabular}




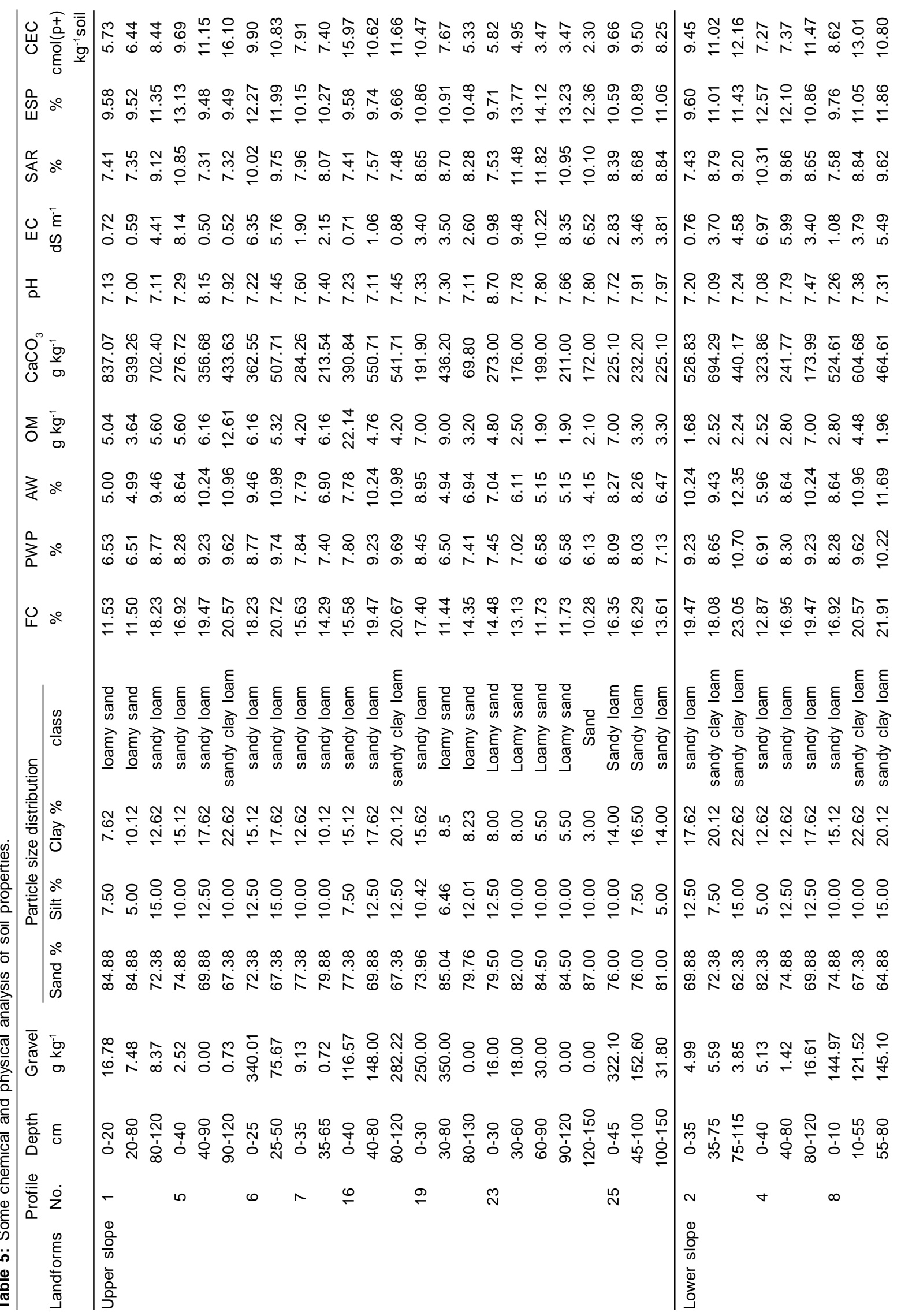




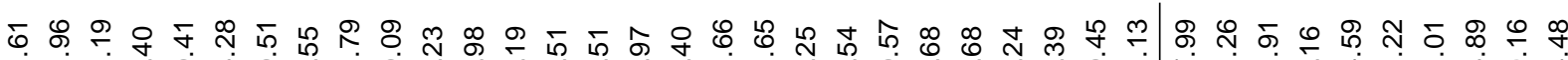

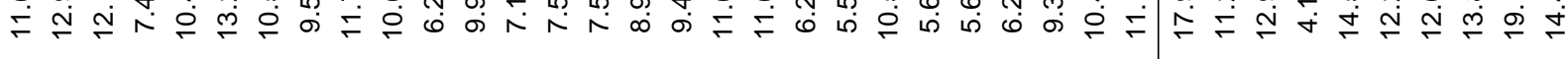

ำ ำ 유 बं

苞

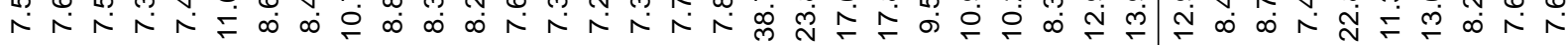

б

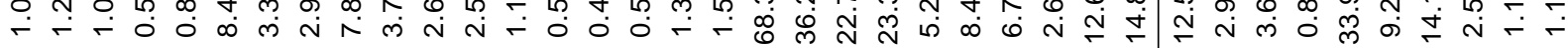
๙

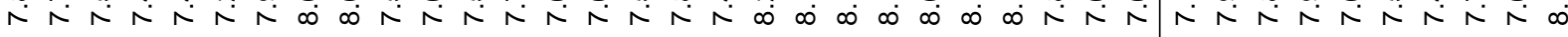

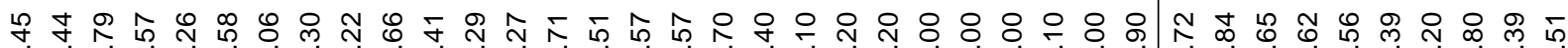
卢

이유 으

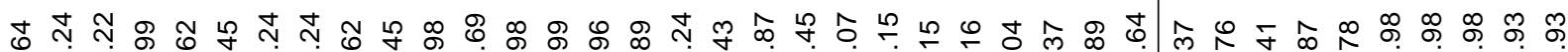

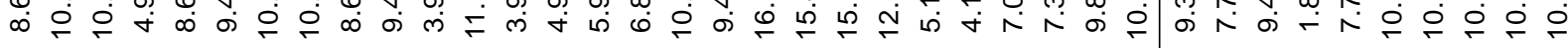

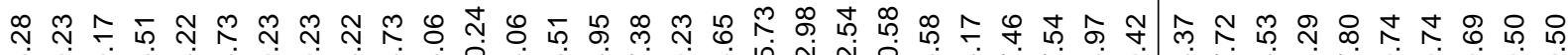

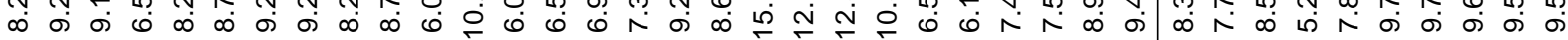

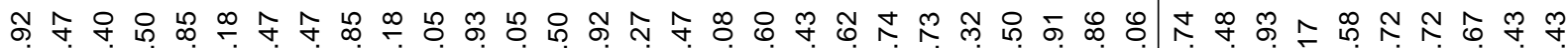

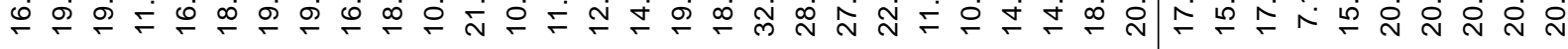

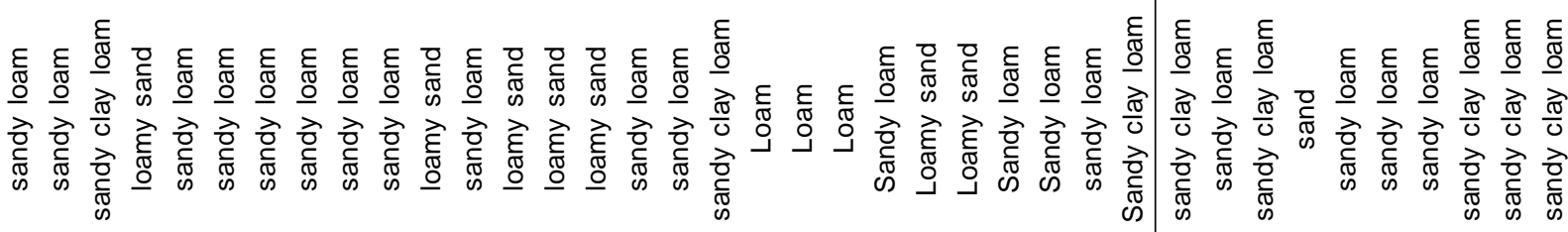

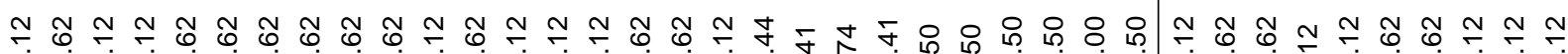

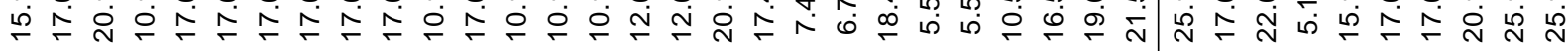

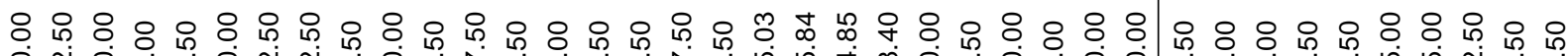
은 은

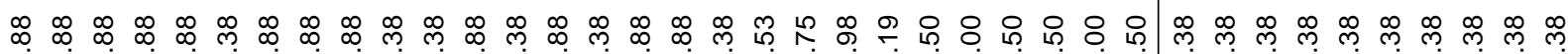
ホं \& ம

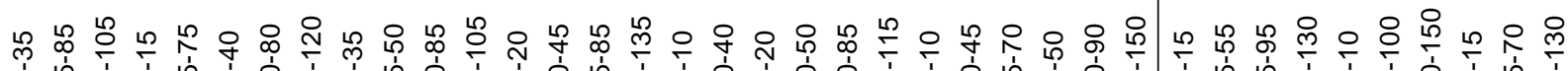

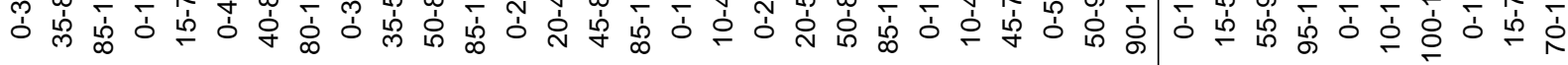

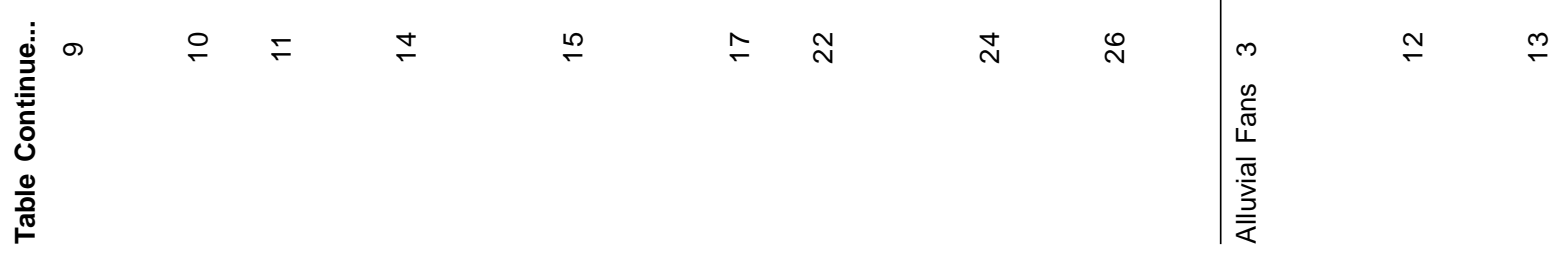




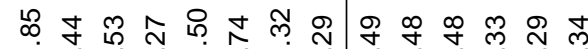
$F$ ம б

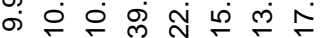

๓

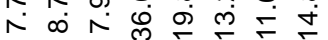

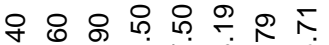
-

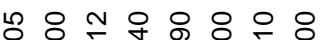

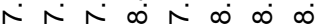

구 8 ○

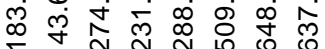
ำ के के के के மூల

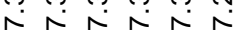

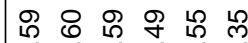
$\begin{array}{llllll}0 & 0 & 0 & 0 & 0 & 0 \\ 0 & 0 & 0 & 0 & 0\end{array}$

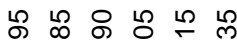
$\sim \wedge \sim$ 으 수 운 으 수 운.

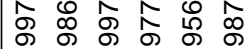

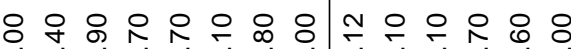

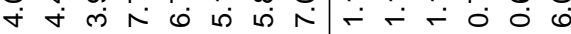

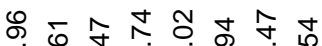
$\bar{E} \dot{0}$ ம

बु

ช 입

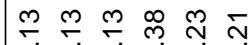

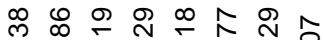

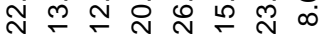

กิก กิก กับ

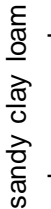

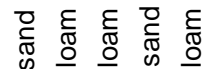
ते $\vec{\imath}$ 하 $\overrightarrow{0}$ त

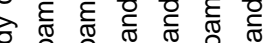

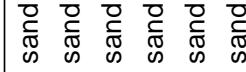
๒ூ

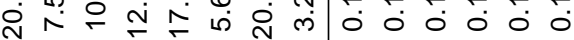
L ம்

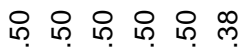

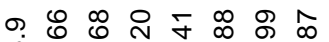
ஸे வे

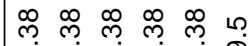

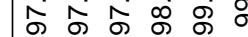

৪ ৪ ப่ 茪

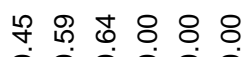

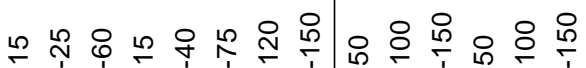

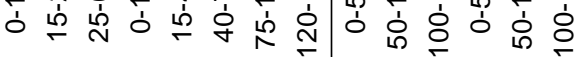

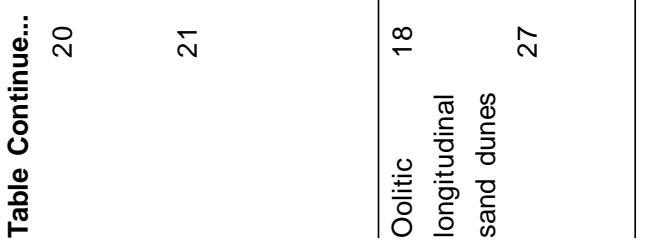

very deep soil except profile 6 and 7 which have moderately depth with 50 and $65 \mathrm{~cm}$ respectively. EC ranged between 0.59 and $10.22 \mathrm{dSm}^{-1}$. Calcium carbonate $\left(\mathrm{CaCO}_{3}\right)$ content was to the tune of $939.26 \mathrm{~g} \mathrm{~kg}^{-1}$ and $93.93 \%$ the soils were considered as extremely calcareous. The soils were classified as Typic Torripsamments (12.50\%), Typic Haplocalcids (25\%), Typic Torriorthents (37.50\%) and Lithic Torriorthents (25\%) as shown in Table 6.

\section{Soils of lower slope unit}

This unit is the largest unit and located in the middle part of the area and occupies an area of $99.88 \mathrm{~km}^{2}$ and represented by twelve soil profiles (Table 5 ). Soil depth varied from shallow to very deep (40 to $150 \mathrm{~cm}$ ). EC values ranged between 0.44 and $14.81 \mathrm{dSm}^{-1}$ except soil profile 22 with $E C$ value of $68.3 \mathrm{dSm}^{-1}$ (extremely saline). Soils are extremely calcareous where calcium carbonate reached up to $913.57 \mathrm{~g} \mathrm{~kg}^{-1}(91.36 \%)$. Soils of this unit were classified as Typic Haplocalcids cover (50\%), Typic Torriorthents cover $(33.34 \%)$, Lithic Torriorthents cover $(8.33 \%)$ and Calcic Haplosalids cover (8.33\%) as illustrated in Table 6.

\section{Soils of alluvial fans unit}

This unit is located in the northern part of the area covers (26.07 $\mathrm{km}^{2}$ ) and represented by five soil profiles (Table 5). Most of soils are very deep soil except profile 20 which is shallow $(60$ $\mathrm{cm}$ ). EC ranged between 0.8 and $62.50 \mathrm{dSm}^{-1} . \mathrm{CaCO}_{3}$ content reached up to $860.62 \mathrm{~g} \mathrm{~kg}^{-1}(86.06 \%)$. Soils of this unit were classified as Typic Haplosalids (20\%), Typic Torriorthents (40\%) and Typic Haplocalcids (40\%) as shown in Table 6.

\section{Soils of oolitic longitudinal sand dunes unit}

This unit is located in the northern part of the investigated area parallel with shore line. It occupies an area of $3.32 \mathrm{~km}^{2}$ and represented by two soil profiles (Table 5). Soils of this unit classified as a very deep soil. EC values varied between 0.46 and $0.60 \mathrm{dSm}^{-1}$. $\mathrm{CaCO}_{3}$ content reached up to 997.50 $\mathrm{g} \mathrm{kg}^{-1}(99.75 \%)$. Soils of this unit were classified as Typic Torripsamments (Table 6).

\section{Land Capability of investigated soils Modified Storie Index}

The area could be classified into four capability classes (Fig 5; Table 7). Grade 3 occupied an area of $98.98 \mathrm{~km}^{2}(50.19 \%)$ while grade 4 had an area of $58.57 \mathrm{~km}^{2}(29.70 \%)$ as illustrated in Table 8 . The common limiting factors are soil salinity, shallow soil depth and coarse of texture class.

\section{MicroLEIS Cervatana model}

The studied area could be classified into three capability classes viz S2, S3 and N (Fig 6; Table 10). Lands with good capability (S2) have a topographic or climatic limitation which in turn restrict the choice for possible crops and their productivity. Land capability (S3) having the limitations of topographic or climatic factors cause limit of potential crops capability of productivity. S3 class includes three sub capability classes S3Ir, S3r and S3I. Marginal land (N) as non-productive land is not recommended for cultivation and may be used for a 
Contribution of Different Land Evaluation Systems to Assess Land Capability and Suitability of Some Coastal Soils in Egypt

Table 6: Legend of the physiographic soil map of the study area.

\begin{tabular}{|c|c|c|c|c|c|c|}
\hline Landform & Area $\mathrm{Km}^{2}$ & $\%$ & Main Soils & $\begin{array}{c}\% \text { of } \\
\text { mapping unit }\end{array}$ & $\begin{array}{l}\text { Represented } \\
\text { Profiles }\end{array}$ & $\begin{array}{c}\text { Kind of } \\
\text { mapping unit }\end{array}$ \\
\hline \multirow[t]{4}{*}{ Upper slope } & 51.77 & 26.25 & Typic Torripsamments & 12.50 & 23 & Complex \\
\hline & & & Typic Torriorthents & 37.50 & $5,16,25$ & \\
\hline & & & Typic Haplocalcids & 25 & 1,19 & \\
\hline & & & Lithic Torriorthents & 25 & 6,7 & \\
\hline \multirow[t]{4}{*}{ Lower slope } & 99.98 & 50.70 & Typic Haplocalcids & 50 & $2,4,8,14,15,24$ & Association \\
\hline & & & Typic Torriorthents & 33.34 & $9,10,11,26$ & \\
\hline & & & Lithic Torriorthents & 8.33 & 17 & \\
\hline & & & Calcic Haplosalids & 8.33 & 22 & \\
\hline \multirow[t]{3}{*}{ Alluvial fans } & 26.07 & 13.22 & Typic Haplosalids & 20 & 21 & \\
\hline & & & Typic Torriorthents & 40 & 3,12 & \\
\hline & & & Typic Haplocalcids & 40 & 13,20 & \\
\hline $\begin{array}{l}\text { Oolitic longitu } \\
\text { dinals and dunes }\end{array}$ & 3.32 & 1.68 & Typic Torripsamments & 100 & 18,27 & Consociation \\
\hline Build-up & 2.60 & 1.32 & No data & - & - & Miscellaneous \\
\hline $\begin{array}{l}\text { Lagoonal depression } \\
\text { and salt marsh }\end{array}$ & 4.61 & 2.34 & No data & - & - & Miscellaneous \\
\hline Oolitic Limestone & 5.17 & 2.62 & No data & - & - & Miscellaneous \\
\hline Oolitic sand beach & 3.70 & 1.88 & No data & - & - & Miscellaneous \\
\hline Total & 197.22 & 100 & & & & \\
\hline
\end{tabular}

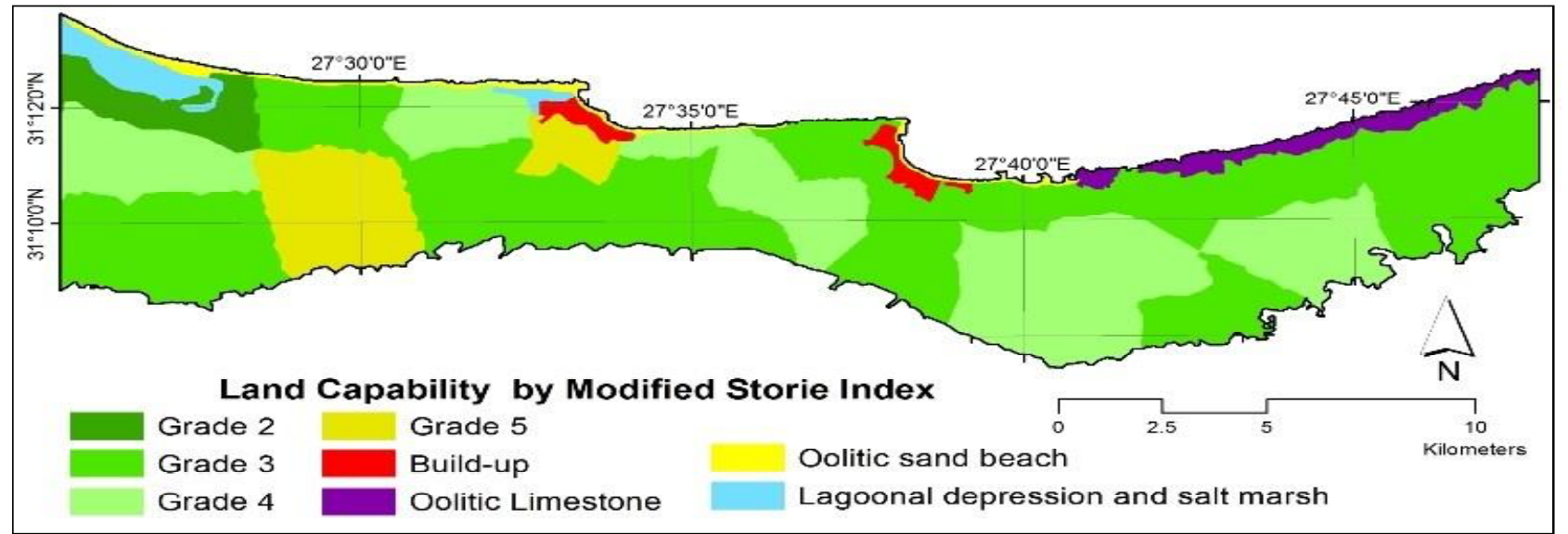

Fig 5: Land capability assessment by Modified Storie Index.

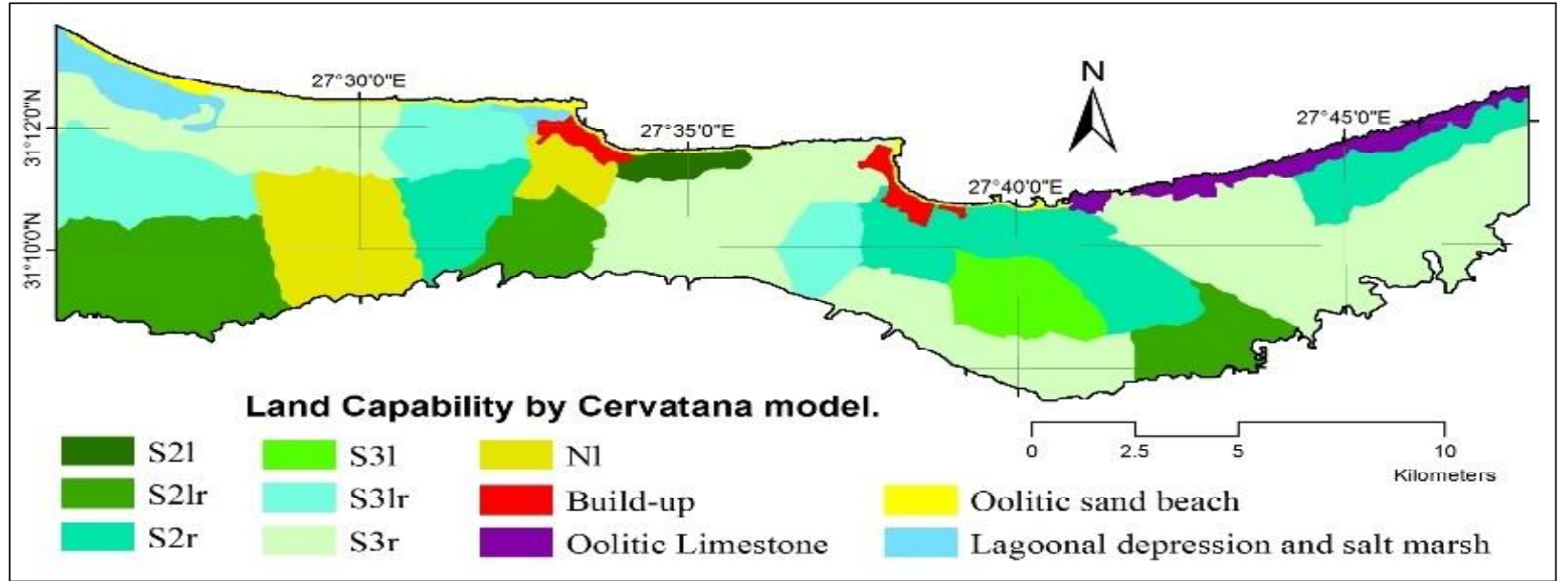

Fig 6: Land capability assessment by MicroLEIS Cervatana model. 
Contribution of Different Land Evaluation Systems to Assess Land Capability and Suitability of Some Coastal Soils in Egypt

Table 7: Land capability by modified Storie index.

\begin{tabular}{|c|c|c|c|c|c|c|c|c|c|c|}
\hline Landforms & P.N. & RateDepth & Rategravel & RateSlope & RatepH & RateSAR & RateEC & Ratetexture & Finalrate & Class \\
\hline \multirow[t]{8}{*}{ Upper slope } & 1 & 84.0 & 99.1 & 89.9 & 100.0 & 87.9 & 92.6 & 80.0 & 48.73 & Grade 3 \\
\hline & 5 & 84.0 & 99.9 & 95.1 & 100.0 & 87.1 & 88.1 & 95.0 & 58.18 & Grade 3 \\
\hline & 6 & 44.1 & 81.5 & 91.0 & 100.0 & 85.1 & 76.7 & 95.0 & 20.28 & Grade 4 \\
\hline & 7 & 54.8 & 99.5 & 93.6 & 100.0 & 87.8 & 92.1 & 95.0 & 39.17 & Grade 4 \\
\hline & 16 & 84.0 & 83.7 & 96.4 & 100.0 & 88.5 & 96.5 & 95.0 & 54.98 & Grade 3 \\
\hline & 19 & 87.6 & 82.8 & 89.8 & 100.0 & 87.0 & 87.8 & 95.0 & 47.28 & Grade 3 \\
\hline & 23 & 93.3 & 98.8 & 91.0 & 100.0 & 84.4 & 72.8 & 80.0 & 41.26 & Grade 3 \\
\hline & 25 & 93.3 & 85.3 & 94.9 & 100.0 & 86.9 & 86.8 & 95.0 & 54.09 & Grade 3 \\
\hline \multirow[t]{12}{*}{ Lower slope } & 2 & 82.0 & 99.6 & 92.0 & 100.0 & 87.1 & 87.9 & 95.0 & 54.55 & Grade 3 \\
\hline & 4 & 36.3 & 99.3 & 89.8 & 100.0 & 85.5 & 79.0 & 95.0 & 20.77 & Grade 4 \\
\hline & 8 & 64.3 & 88.0 & 100.0 & 100.0 & 86.5 & 84.5 & 95.0 & 39.32 & Grade 4 \\
\hline & 9 & 77.6 & 96.2 & 94.9 & 100.0 & 88.4 & 95.6 & 95.0 & 56.83 & Grade 3 \\
\hline & 10 & 61.3 & 98.7 & 100.0 & 100.0 & 88.6 & 97.0 & 80.0 & 41.61 & Grade 3 \\
\hline & 11 & 84.0 & 98.0 & 94.9 & 100.0 & 85.9 & 80.9 & 95.0 & 51.58 & Grade 3 \\
\hline & 14 & 77.6 & 92.7 & 94.9 & 100.0 & 86.1 & 82.5 & 95.0 & 46.07 & Grade 3 \\
\hline & 15 & 89.2 & 97.2 & 96.4 & 100.0 & 88.7 & 97.6 & 80.0 & 57.87 & Grade 3 \\
\hline & 17 & 36.3 & 91.5 & 91.0 & 100.0 & 88.1 & 94.1 & 95.0 & 23.80 & Grade 4 \\
\hline & 22 & 82.0 & 100.0 & 96.4 & 100.0 & 68.7 & 10.6 & 80.0 & 4.59 & Grade 5 \\
\hline & 24 & 58.1 & 93.3 & 94.9 & 100.0 & 84.3 & 71.9 & 80.0 & 24.93 & Grade 4 \\
\hline & 26 & 93.3 & 81.8 & 94.9 & 100.0 & 82.5 & 61.8 & 95.0 & 35.09 & Grade 4 \\
\hline \multirow[t]{5}{*}{ Alluvial fans } & 3 & 87.6 & 99.8 & 91.0 & 100.0 & 86.7 & 85.6 & 95.0 & 56.05 & Grade 3 \\
\hline & 12 & 93.3 & 100.0 & 98.4 & 100.0 & 81.0 & 53.7 & 95.0 & 37.91 & Grade 4 \\
\hline & 13 & 87.6 & 99.9 & 93.6 & 100.0 & 88.2 & 94.8 & 95.0 & 65.03 & Grade 2 \\
\hline & 20 & 51.4 & 75.5 & 94.2 & 100.0 & 87.8 & 91.9 & 95.0 & 28.00 & Grade 4 \\
\hline & 21 & 93.3 & 100.0 & 96.4 & 100.0 & 76.4 & 28.7 & 95.0 & 18.72 & Grade 5 \\
\hline \multirow{2}{*}{$\begin{array}{l}\text { Oolitic longitudinal } \\
\text { sand dunes }\end{array}$} & 18 & 93.3 & 99.9 & 95.4 & 100.0 & 88.7 & 97.6 & 60.0 & 46.26 & Grade 3 \\
\hline & 27 & 93.3 & 100.0 & 92.0 & 100.0 & 88.8 & 98.2 & 60.0 & 44.89 & Grade 3 \\
\hline
\end{tabular}

Table 8: Tabulate area between Storie capability and landforms $\mathrm{km}^{2}$.

\begin{tabular}{lccccc}
\hline Landform & Upper slope & Lower slope & Alluvial fans & Oolitic longitudinal sand dunes & Total \\
\hline Grade 2 & 0.00 & 0.82 & 6.32 & 0.00 & 7.13 \\
Grade 3 & 29.79 & 54.78 & 11.63 & 2.79 & 98.98 \\
Grade 4 & 17.92 & 33.15 & 6.98 & 0.53 & 58.57 \\
Grade 5 & 4.06 & 11.24 & 1.15 & 0.00 & 16.45 \\
Total & 51.77 & 99.98 & 26.07 & 3.32 & 181.14 \\
\hline
\end{tabular}

Table 9: Tabulate area between MicroLEIS capability and land form $\mathrm{km}^{2}$.

\begin{tabular}{|c|c|c|c|c|c|c|}
\hline \multicolumn{2}{|c|}{ Capability } & \multirow{2}{*}{ Upper slope } & \multirow{2}{*}{ Lower slope } & \multirow{2}{*}{ Alluvial fans } & \multirow{2}{*}{$\begin{array}{l}\text { Oolitic longitudinal } \\
\text { sand dunes }\end{array}$} & \multirow{2}{*}{ Total } \\
\hline Class & Subclass & & & & & \\
\hline \multirow[t]{3}{*}{ S2 } & S2lr & 17.91 & 11.36 & 0.00 & 0.00 & 29.27 \\
\hline & $s 2 r$ & 3.04 & 22.94 & 2.50 & 0.97 & 29.45 \\
\hline & S2I & 0.00 & 0.67 & 1.48 & 0.00 & 2.15 \\
\hline \multirow[t]{3}{*}{ S3 } & S3r & 25.90 & 32.48 & 15.45 & 1.83 & 75.65 \\
\hline & S3I & 0.74 & 6.62 & 0.00 & 0.00 & 7.36 \\
\hline & S3Ir & 0.11 & 14.68 & 5.50 & 0.53 & 20.81 \\
\hline \multirow[t]{2}{*}{$N$} & $\mathrm{NI}$ & 4.06 & 11.24 & 1.15 & 0.00 & 16.45 \\
\hline & & 26.07 & 3.32 & 51.77 & 99.98 & 181.14 \\
\hline
\end{tabular}

Good . S2 ; Moderate, S3; Marginal, N ; Soil, I ; Erosion risks, r. 
Table 10: Suitability by Almagra model.

\begin{tabular}{|c|c|c|c|c|c|c|c|c|c|c|c|c|c|}
\hline Landform & P.N & $\mathrm{T}$ & $\mathrm{M}$ & $\mathrm{Me}$ & $\mathrm{P}$ & $S$ & $A$ & $\mathrm{G}$ & Af & $\mathrm{Me}$ & $\mathrm{C}$ & $\mathrm{O}$ & $\begin{array}{c}\text { Land } \\
\text { capability }\end{array}$ \\
\hline \multirow[t]{8}{*}{ Upper slope } & 1 & $S 4 t$ & $S 4 t$ & $S 4 t$ & S4tc & $S 4 t$ & $\mathrm{~S} 4 \mathrm{t}$ & $S 4 t$ & $S 4 t$ & $S 4 c$ & S4c & S3c & S2r \\
\hline & 5 & S3t & S3tc & S3tc & S3tc & S3t & S3tc & S3t & S3t & $\mathrm{s} 3 \mathrm{c}$ & $\mathrm{s} 3 \mathrm{c}$ & S2tcs & S2Ir \\
\hline & 6 & $S 4 t$ & $\mathrm{~S} 4 \mathrm{t}$ & S4t & S4t & $S 4 t$ & $\mathrm{~s} 4 \mathrm{t}$ & S4t & S4t & S3tcs & S3tcs & S3ts & $\mathrm{S} 3 \mathrm{r}$ \\
\hline & 7 & S3t & S3t & S3t & S3t & S3t & S3t & S3t & S3t & S2tca & S2tca & S2ta & $S 3 r$ \\
\hline & 16 & $S 4 t$ & $S 4 t$ & $S 4 t$ & $S 4 t$ & $S 4 t$ & $S 4 t$ & $S 4 t$ & $S 4 t$ & S3tc & S3tc & S3t & S2Ir \\
\hline & 19 & $S 4 t$ & $S 4 t$ & $S 4 t$ & $S 4 t$ & $S 4 t$ & $S 4 t$ & S4t & S4t & s3t & S3t & S3t & $S 3 r$ \\
\hline & 23 & $S 4 t$ & $S 4 t$ & $\mathrm{~S} 4 \mathrm{t}$ & $\mathrm{S} 4 \mathrm{t}$ & $\mathrm{S} 4 \mathrm{t}$ & $\mathrm{S} 4 \mathrm{t}$ & $\mathrm{S} 4 \mathrm{t}$ & $\mathrm{S} 4 \mathrm{t}$ & S4s & S4s & S3s & S2Ir \\
\hline & 25 & S4ta & S5a & S4ta & S4ta & S4ta & S4ta & S4ta & S4ta & S4a & $\mathrm{S} 4 \mathrm{a}$ & $\mathrm{S} 4 \mathrm{a}$ & $\mathrm{S} 3 \mathrm{r}$ \\
\hline \multirow[t]{12}{*}{ Lower slope } & 2 & S3tc & S3tc & S3tc & S4c & S3tc & S3tc & S3tc & S3tc & S4c & S4c & S3c & S2r \\
\hline & 4 & S3td & S3ta & S3ts & S3t & S3td & S3t & S3t & S3td & S4d & S4d & S4d & S3Ir \\
\hline & 8 & S3tc & S3tca & S3tc & $\mathrm{S} 4 \mathrm{c}$ & S3tc & S3tc & S3tc & S3tc & $\mathrm{S} 4 \mathrm{c}$ & $S 4 c$ & S3c & $S 2 r$ \\
\hline & 9 & S3t & S3tc & S3tc & S3tc & S3t & S3tc & S3t & S3t & S3c & S3c & S2tca & s2r \\
\hline & 10 & S3tc & S3tc & S3tc & $\mathrm{S} 4 \mathrm{c}$ & S3tc & S3tc & S3tc & S3tc & $\mathrm{S} 4 \mathrm{c}$ & $\mathrm{S} 4 \mathrm{c}$ & S3c & S3r \\
\hline & 11 & S3t & S3tca & S3tcs & S3tc & S3t & S3tc & S3t & S3t & S3cs & S3cs & S3s & s3r \\
\hline & 14 & S3t & S3tca & S3tc & S3tc & S3t & S3tc & S3t & S3t & $\mathrm{S} 3 \mathrm{c}$ & $\mathrm{S} 3 \mathrm{c}$ & S2tcs & $\mathrm{s} 3 \mathrm{r}$ \\
\hline & 15 & $\mathrm{~S} 4 \mathrm{t}$ & S4t & $\mathrm{S} 4 \mathrm{t}$ & S4tc & $\mathrm{S} 4 \mathrm{t}$ & $\mathrm{S} 4 \mathrm{t}$ & $S 4 t$ & $\mathrm{~S} 4 \mathrm{t}$ & $\mathrm{S} 4 \mathrm{c}$ & S4c & S3c & $\mathrm{S} 2 \mathrm{r}$ \\
\hline & 17 & S3td & S3tc & S3tc & S3tc & S3td & S3tc & S3t & S3td & S4d & S4d & S4d & S3Ir \\
\hline & 22 & S5s & S5s & S5s & S5s & S5s & S5s & S5s & S5s & S5s & S5s & S5s & $\mathrm{NI}$ \\
\hline & 24 & S4t & $S 4 t$ & $S 4 t$ & $S 4 t$ & $S 4 t$ & $S 4 t$ & S4t & $S 4 t$ & $\mathrm{~S} 4 \mathrm{~s}$ & $\mathrm{~S} 4 \mathrm{~s}$ & S3s & $\mathrm{S} 3 \mathrm{r}$ \\
\hline & 26 & S5a & $\mathrm{S} 5 \mathrm{a}$ & S5a & $\mathrm{S} 5 \mathrm{a}$ & S5a & S5a & $\mathrm{S} 5 \mathrm{a}$ & S5a & S5sa & S5sa & S5a & S3l \\
\hline \multirow[t]{5}{*}{ Alluvial Fans } & 3 & S3c & S3ca & S3c & S4c & S3c & S3c & S3c & S3c & S4c & S4c & S3c & S3r \\
\hline & 12 & $\mathrm{~S} 5 \mathrm{~s}$ & $\mathrm{~S} 5 \mathrm{~s}$ & S5s & S5s & S5s & $\mathrm{S} 4 \mathrm{~s}$ & $\mathrm{~S} 5 \mathrm{~s}$ & $\mathrm{~S} 4 \mathrm{~s}$ & S5s & S5s & $\mathrm{S} 5 \mathrm{~s}$ & S3Ir \\
\hline & 13 & S2ca & $\mathrm{s} 3 \mathrm{c}$ & $\mathrm{s} 3 \mathrm{c}$ & S3c & S2ca & S3c & S2ca & S2ca & S3c & s3c & S2tca & $s 3 r$ \\
\hline & 20 & S2t & $\mathrm{S} 2 \mathrm{tc}$ & S3t & S3t & S2t & S3t & S2t & $\mathrm{S} 2 \mathrm{t}$ & S3t & S3t & $\mathrm{S} 4 \mathrm{t}$ & S2l \\
\hline & 21 & S5s & S5s & S5s & S5s & S5s & S5s & S5s & S5s & S5s & S5s & S5s & $\mathrm{NI}$ \\
\hline Oolitic longitudi- & 18 & S4t & S4t & $S 4 t$ & S4tc & S4t & S4t & S4t & S4t & S4c & S4c & S3c & S3r \\
\hline -nal sand dunes & 27 & $S 4 t$ & S4ta & $S 4 t$ & S4tc & $S 4 t$ & $S 4 t$ & S4t & $S 4 t$ & $\mathrm{~S} 4 \mathrm{c}$ & $S 4 c$ & S3ca & S3r \\
\hline
\end{tabular}

maize (M), melon (Me), potato $(P)$, soybean $(S)$, wheat $(T)$, cotton $(A)$, sunflower $(G)$, alfalfa (Af), citrus $(C)$, peach (Me), olive $(O)$. optimum suitable (S1), high suitable (S2), moderate suitable (S3), marginal suitable (S4), not suitable (S5), Useful depth (p), Texture (t), Drainage $(\mathrm{d})$, Carbonate(c), Salinity $(\mathrm{s})$, Sodium saturation (a), Profile development $(\mathrm{g})$, Slope $(\mathrm{t})$, Soil (I), Erosion risks ( $\mathrm{r}$ ), Bioclimatic deficit (b).

pasture or forestry. NI sub-class occupied an area of 16.45 $\mathrm{km}^{2}(8.34 \%)$ and affected by salinity and soil depth and some physical limitations (Table 9).

\section{Land suitability assessment}

\section{MicroLEIS Almagra model}

The investigated soils are classified into four suitability classes vis high suitable (S2), moderate suitable (S3), marginal suitable (S4) and not suitable (S5). Land suitability analysis indicated that $4.71 \%$ of the studied area is S2 for wheat, soya, sunflower, alfalfa and citrus whereas $14.82 \%$ of the study area is S2 for olives (Table 11). The common limitations in theses soils are calcium carbonate, salinity and soil texture. About $31.78 \%$ of the study area is S3 for wheat, maize, peach, citrus, cotton, sunflower and alfalfa. About $36.5 \%$ of the study area is S3 for watermelon and about $52 \%$ of the study area is S3 for olives. About $40 \%$ is S4 for most of crops evaluated. Soil salinity, excess of calcium carbonate, drainage and soil texture were the most common limiting factors in these soils (Fig 7; Table10).

\section{LUST model}

The investigated soils are classified into three suitability classes as highly suitable (S1), moderately suitable (S2) and marginally suitable (S3) (Table 12; Fig 8). Land suitability analysis indicated that $5.85,3.73$ and $2.11 \%$ of the area are $\mathrm{S} 1$ for wheat, cotton and olives respectively (Table 13). About $31 \%$ of the study area is S2 for citrus, peach and soya where $63.86 \%$ is moderately suitable for alfalfa and sunflower. About $85 \%$ of the area is moderately suitable for wheat and melon. Finally, about $55 \%$ of the study area is moderately suitable for potato and cotton while $75 \%$ is moderately suitable for maize and olives. About $30 \%$ of the area is S3 for most of the selected crops (Table 13). Soil salinity, excess of calcium carbonate, drainage and soil texture were the most common limiting factors in the studied soils.

\section{CONCLUSION}

It may be concluded that Storie Index categorized $50 \%$ of the area as S3. Cervatana model showed that $52.5 \%$ of the 
Contribution of Different Land Evaluation Systems to Assess Land Capability and Suitability of Some Coastal Soils in Egypt

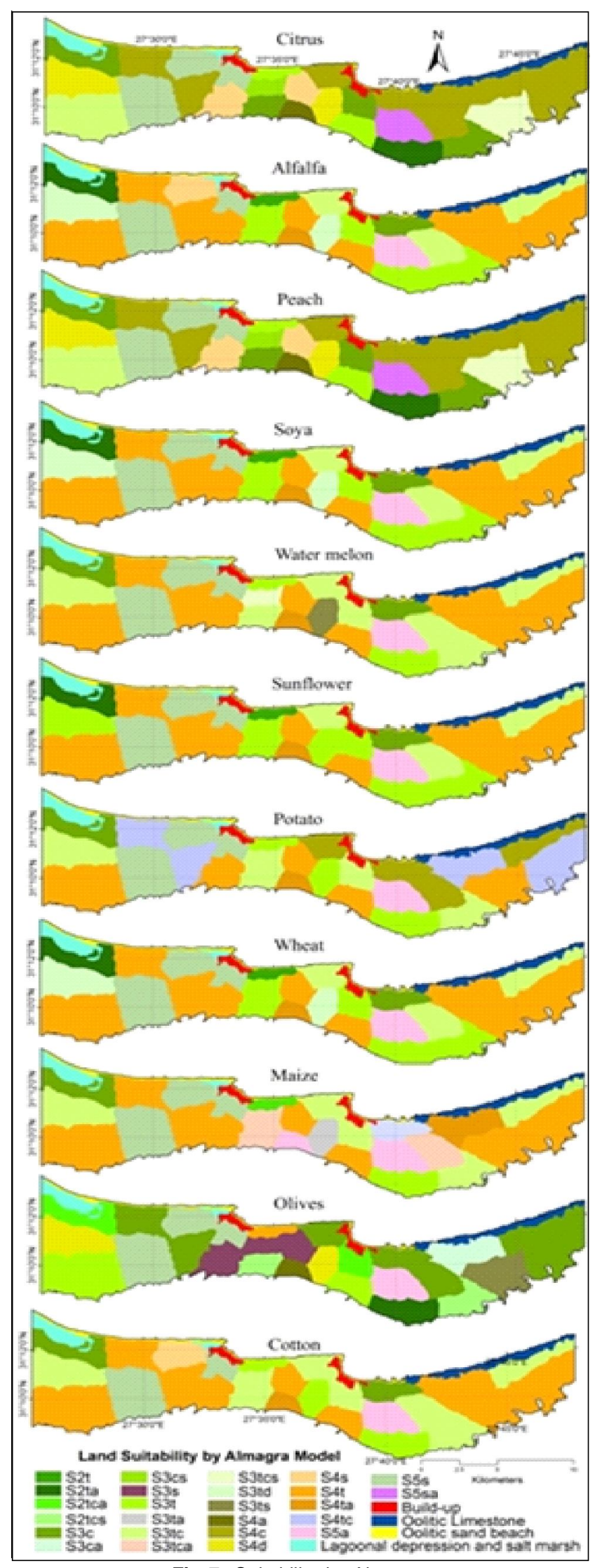

Fig 7: Suitability by Almagra.

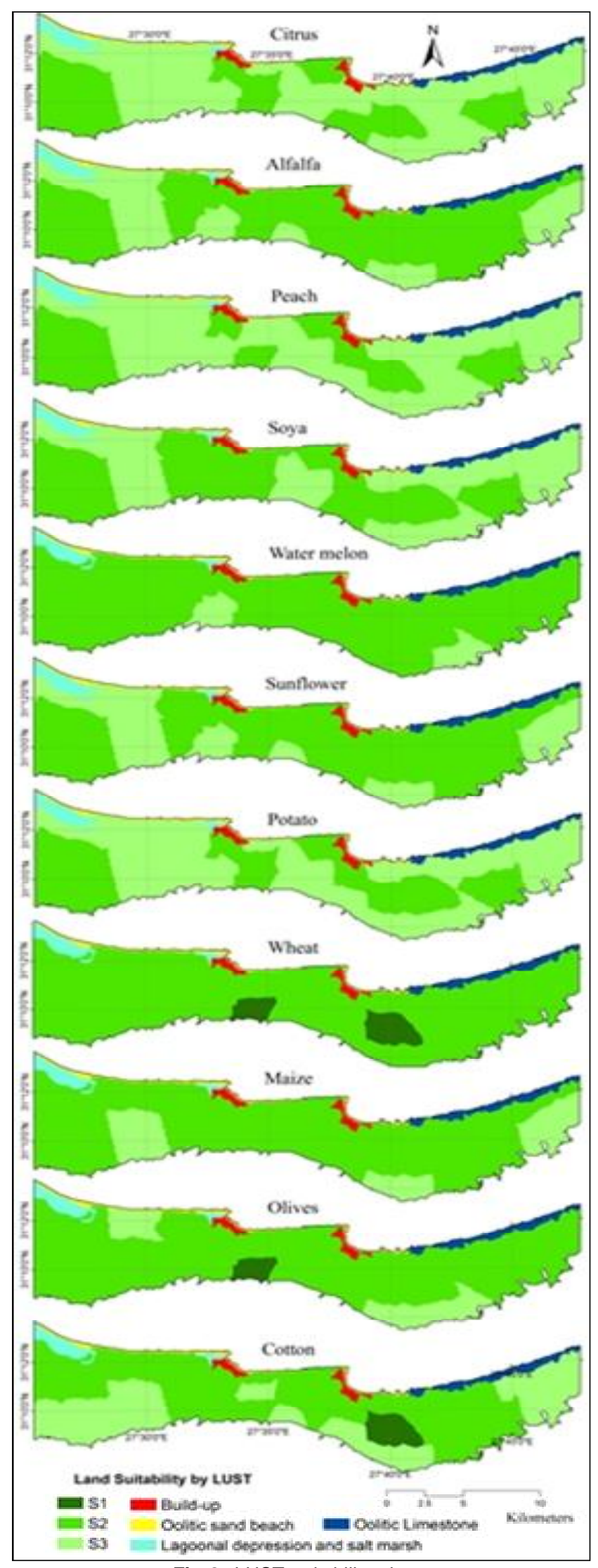

Fig 8: LUST suitability class. 
Contribution of Different Land Evaluation Systems to Assess Land Capability and Suitability of Some Coastal Soils in Egypt

Table 11: Tabulate area in $\mathrm{km}^{2}$ between Microlies suitability and land form.

\begin{tabular}{|c|c|c|c|c|c|c|}
\hline \multirow[b]{2}{*}{ Crop } & \multirow[b]{2}{*}{ Class } & \multicolumn{4}{|c|}{ Landform } & \multirow[b]{2}{*}{ Total } \\
\hline & & $\begin{array}{l}\text { Upper } \\
\text { slope }\end{array}$ & $\begin{array}{l}\text { Lower } \\
\text { slope }\end{array}$ & $\begin{array}{c}\text { Alluvial } \\
\text { fans }\end{array}$ & $\begin{array}{l}\text { Oolitic longitudinal } \\
\text { sand dunes }\end{array}$ & \\
\hline \multirow[t]{4}{*}{ Wheat } & $\mathrm{S} 2$ & 0.00 & 1.48 & 7.80 & 0.00 & 9.28 \\
\hline & S3 & 16.35 & 40.83 & 3.72 & 1.81 & 62.71 \\
\hline & S4 & 30.62 & 39.63 & 7.94 & 0.98 & 79.17 \\
\hline & S5 & 4.80 & 18.03 & 6.62 & 0.53 & 29.98 \\
\hline \multirow[t]{4}{*}{ Maize } & $\mathrm{S} 2$ & 0.00 & 0.67 & 1.48 & 0.00 & 2.15 \\
\hline & S3 & 16.35 & 41.65 & 10.04 & 1.81 & 69.85 \\
\hline & S4 & 30.08 & 37.47 & 7.94 & 0.98 & 76.46 \\
\hline & S5 & 5.34 & 20.19 & 6.62 & 0.53 & 32.68 \\
\hline \multirow[t]{3}{*}{ Melon } & S3 & 16.35 & 42.32 & 11.52 & 1.81 & 71.99 \\
\hline & S4 & 30.62 & 39.63 & 7.94 & 0.98 & 79.17 \\
\hline & S5 & 4.80 & 18.03 & 6.62 & 0.53 & 29.98 \\
\hline \multirow[t]{3}{*}{ Potato } & S3 & 14.88 & 26.04 & 8.18 & 0.00 & 49.10 \\
\hline & S4 & 32.08 & 55.91 & 11.28 & 2.79 & 102.07 \\
\hline & S5 & 4.80 & 18.03 & 6.62 & 0.53 & 29.98 \\
\hline \multirow[t]{4}{*}{ Soya } & $\mathrm{S} 2$ & 0.00 & 1.48 & 7.80 & 0.00 & 9.28 \\
\hline & S3 & 16.35 & 40.83 & 3.72 & 1.81 & 62.71 \\
\hline & S4 & 30.62 & 39.63 & 7.94 & 0.98 & 79.17 \\
\hline & S5 & 4.80 & 18.03 & 6.62 & 0.53 & 29.98 \\
\hline \multirow[t]{3}{*}{ Cotton } & S3 & 16.35 & 42.32 & 11.52 & 1.81 & 71.99 \\
\hline & S4 & 30.62 & 39.80 & 13.41 & 1.50 & 85.34 \\
\hline & S5 & 4.80 & 17.86 & 1.15 & 0.00 & 23.81 \\
\hline \multirow[t]{4}{*}{ Sunflower } & $\mathrm{S} 2$ & 0.00 & 1.48 & 7.80 & 0.00 & 9.28 \\
\hline & S3 & 16.35 & 40.83 & 3.72 & 1.81 & 62.71 \\
\hline & S4 & 30.62 & 39.63 & 7.94 & 0.98 & 79.17 \\
\hline & S5 & 4.80 & 18.03 & 6.62 & 0.53 & 29.98 \\
\hline \multirow[t]{4}{*}{ Alfaalfa } & S2 & 0.00 & 1.48 & 7.80 & 0.00 & 9.28 \\
\hline & S3 & 16.35 & 40.83 & 3.72 & 1.81 & 62.71 \\
\hline & S4 & 30.62 & 39.80 & 13.41 & 1.50 & 85.34 \\
\hline & S5 & 4.80 & 17.86 & 1.15 & 0.00 & 23.81 \\
\hline \multirow[t]{4}{*}{ Peach } & $\mathrm{S} 2$ & 6.93 & 0.24 & 0.00 & 0.00 & 7.18 \\
\hline & S3 & 27.45 & 23.40 & 8.15 & 0.00 & 59.00 \\
\hline & S4 & 12.58 & 58.31 & 11.30 & 2.79 & 84.99 \\
\hline & S5 & 4.80 & 18.03 & 6.62 & 0.53 & 29.98 \\
\hline \multirow[t]{4}{*}{ Citrus } & $\mathrm{S} 2$ & 6.93 & 0.24 & 0.00 & 0.00 & 7.18 \\
\hline & S3 & 27.45 & 23.40 & 8.15 & 0.00 & 59.00 \\
\hline & S4 & 12.58 & 58.31 & 11.30 & 2.79 & 84.99 \\
\hline & S5 & 4.80 & 18.03 & 6.62 & 0.53 & 29.98 \\
\hline \multirow[t]{4}{*}{ Olives } & $\mathrm{S} 2$ & 14.77 & 7.79 & 6.67 & 0.00 & 29.23 \\
\hline & S3 & 31.54 & 56.82 & 11.28 & 2.79 & 102.43 \\
\hline & S4 & 0.65 & 17.34 & 1.50 & 0.00 & 19.50 \\
\hline & S5 & 4.80 & 18.03 & 6.62 & 0.53 & 29.98 \\
\hline
\end{tabular}

soils were classified as S3. The main land capability limitations were erosion risk, excess of soil salinity and shallow soil depth. Land suitability analysis by Almagra model showed that about $4.71 \%$ and $31.78 \%$ of the area are S2 for wheat and citrus respectively. Olives had an area of $14.82 \%$ and $52 \%$ as S2 and S3 respectively. LUST results showed that about $5.85,3.73$ and $2.11 \%$ of the area are $\mathrm{S} 1$ for wheat, cotton and olives respectively. About $31 \%$ of the study area is S2 for citrus, peach and soya whereas $63.86 \%$ is S2 for alfalfa and sunflower and $85 \%$ of the area is S2 for wheat and melon. The main limitation factors were soil salinity, calcium carbonate, drainage and texture. Thus MicroLEIS application either Cervatana or Almagra to predict land suitability and land capability respectively is not 
Contribution of Different Land Evaluation Systems to Assess Land Capability and Suitability of Some Coastal Soils in Egypt

Table 12: LUST suitability class.

\begin{tabular}{|c|c|c|c|c|c|c|c|c|c|c|c|c|}
\hline Landform & LU & $\mathrm{T}$ & $\mathrm{M}$ & $\mathrm{Me}$ & $\mathrm{P}$ & $S$ & A & $\mathrm{G}$ & Af & $\mathrm{Me}$ & C & 0 \\
\hline \multirow{8}{*}{ Upper slope } & 1 & S2 & S2 & S2 & S3 & S3 & S3 & S2 & S2 & S3 & S3 & $\mathrm{S} 2$ \\
\hline & 5 & S2 & S2 & $\mathrm{S} 2$ & S2 & S2 & S2 & S2 & S2 & $\mathrm{S} 2$ & S2 & S2 \\
\hline & 6 & S2 & S3 & S2 & S3 & S3 & S3 & S3 & S3 & S3 & S3 & S3 \\
\hline & 7 & S2 & S2 & S2 & S2 & S2 & S2 & S2 & S2 & S3 & S3 & $\mathrm{S} 2$ \\
\hline & 16 & S2 & S2 & S2 & S2 & S2 & S2 & S2 & S2 & S2 & S2 & $\mathrm{S} 2$ \\
\hline & 19 & S2 & S2 & $\mathrm{S} 2$ & S3 & $\mathrm{S} 2$ & $\mathrm{~S} 2$ & S2 & $\mathrm{S} 2$ & S3 & S3 & S2 \\
\hline & 23 & S2 & S2 & S2 & S3 & S3 & S2 & S2 & S2 & S3 & S3 & S2 \\
\hline & 25 & S1 & S2 & S2 & S2 & S2 & S1 & S2 & S2 & S2 & S2 & $\mathrm{S} 2$ \\
\hline \multirow[t]{12}{*}{ Lower slope } & 2 & S2 & S2 & S2 & S3 & S3 & S2 & S2 & S2 & S3 & S3 & S2 \\
\hline & 4 & $\mathrm{~S} 2$ & $\mathrm{~S} 2$ & S3 & S3 & S3 & S2 & S2 & $\mathrm{S} 2$ & S3 & S3 & S3 \\
\hline & 8 & S2 & S2 & S2 & S2 & S2 & S2 & S2 & S2 & S2 & S3 & S2 \\
\hline & 9 & S2 & S2 & S2 & S2 & S2 & S2 & S2 & S2 & S2 & S2 & S2 \\
\hline & 10 & S2 & S2 & S2 & S2 & $\mathrm{S} 2$ & S3 & S2 & S2 & S3 & S2 & S2 \\
\hline & 11 & S2 & S2 & S2 & S3 & S2 & S2 & S2 & S2 & S3 & S3 & $\mathrm{S} 2$ \\
\hline & 14 & $\mathrm{~S} 2$ & S2 & $\mathrm{S} 2$ & S3 & $\mathrm{S} 2$ & S2 & S2 & $\mathrm{S} 2$ & S3 & S3 & $\mathrm{S} 2$ \\
\hline & 15 & S2 & S2 & S2 & S2 & S2 & S3 & S2 & S2 & S2 & S2 & S2 \\
\hline & 17 & S2 & $\mathrm{S} 2$ & $\mathrm{~S} 2$ & S3 & S3 & S2 & S3 & S3 & S3 & S3 & S3 \\
\hline & 22 & S2 & S2 & S3 & S3 & $\mathrm{S} 2$ & S2 & S3 & S3 & S3 & S3 & $\mathrm{S} 2$ \\
\hline & 24 & $\mathrm{~S} 2$ & S2 & $\mathrm{S} 2$ & S3 & S3 & S3 & S3 & S3 & S3 & S3 & $\mathrm{S} 2$ \\
\hline & 26 & $\mathrm{~S} 2$ & $\mathrm{~S} 2$ & $\mathrm{~S} 2$ & S3 & S3 & $\mathrm{S} 2$ & S2 & $\mathrm{S} 2$ & S3 & S3 & $\mathrm{S} 2$ \\
\hline \multirow[t]{5}{*}{ Alluvial fans } & 3 & S2 & S2 & S2 & S3 & S3 & S2 & S2 & S2 & S3 & S3 & $\mathrm{S} 2$ \\
\hline & 12 & S2 & S2 & $\mathrm{S} 2$ & S3 & S3 & S2 & S3 & S3 & S3 & S3 & $\mathrm{S} 2$ \\
\hline & 13 & S1 & $\mathrm{S} 2$ & $\mathrm{~S} 2$ & S2 & $\mathrm{S} 2$ & S2 & $\mathrm{S} 2$ & $\mathrm{~S} 2$ & S2 & S2 & S1 \\
\hline & 20 & S2 & S2 & S2 & S2 & S2 & S2 & S2 & S2 & S2 & S2 & $\mathrm{S} 2$ \\
\hline & 21 & $\mathrm{~S} 2$ & S3 & $\mathrm{S} 2$ & S3 & S3 & S3 & S3 & S3 & S3 & S3 & $\mathrm{S} 2$ \\
\hline \multirow{2}{*}{$\begin{array}{l}\text { Oolitic longitudinal } \\
\text { sand dunes }\end{array}$} & 18 & S2 & S2 & S2 & S3 & S2 & S3 & S2 & S2 & S3 & S2 & $\mathrm{S} 2$ \\
\hline & 27 & $\mathrm{~S} 2$ & S3 & $\mathrm{S} 2$ & S3 & S3 & S3 & S3 & S3 & S3 & S3 & $\mathrm{S} 2$ \\
\hline
\end{tabular}

Table 13: Tabulate area in $\mathrm{km}^{2}$ between LUST suitability and landforms.

\begin{tabular}{|c|c|c|c|c|c|c|}
\hline \multirow[b]{2}{*}{ Crop } & \multirow[b]{2}{*}{ Class } & \multicolumn{4}{|c|}{ Landform } & \multirow[b]{2}{*}{ Total } \\
\hline & & $\begin{array}{l}\text { Upper } \\
\text { slope }\end{array}$ & $\begin{array}{l}\text { Lower } \\
\text { slope }\end{array}$ & $\begin{array}{l}\text { Alluvial } \\
\text { fans }\end{array}$ & $\begin{array}{l}\text { Oolitic longitudinal } \\
\text { sand dunes }\end{array}$ & \\
\hline \multirow{2}{*}{ Citrus } & S2 & 21.75 & 39.43 & 2.48 & 0.85 & 64.51 \\
\hline & S3 & 30.01 & 60.55 & 23.59 & 2.47 & 116.63 \\
\hline \multirow{2}{*}{ Alfalfa } & $\mathrm{S} 2$ & 31.57 & 77.89 & 13.72 & 2.77 & 125.95 \\
\hline & S3 & 20.19 & 22.09 & 12.36 & 0.54 & 55.19 \\
\hline \multirow{2}{*}{ Peach } & S2 & 19.96 & 37.18 & 2.83 & 0.85 & 60.82 \\
\hline & S3 & 31.81 & 62.80 & 23.24 & 2.47 & 120.31 \\
\hline \multirow{2}{*}{ Soybean } & $\mathrm{S} 2$ & 27.14 & 58.52 & 9.90 & 1.37 & 96.94 \\
\hline & S3 & 24.62 & 41.46 & 16.17 & 1.94 & 84.20 \\
\hline \multirow{2}{*}{ Watermelon } & S2 & 42.99 & 96.30 & 26.07 & 3.32 & 168.68 \\
\hline & S3 & 8.78 & 3.68 & 0.00 & 0.00 & 12.46 \\
\hline \multirow{2}{*}{ Sunflower } & $\mathrm{S} 2$ & 31.57 & 77.89 & 13.72 & 2.77 & 125.95 \\
\hline & S3 & 20.19 & 22.09 & 12.36 & 0.54 & 55.19 \\
\hline \multirow{3}{*}{ Potato } & $\mathrm{S} 2$ & 30.35 & 54.30 & 23.24 & 2.47 & 110.35 \\
\hline & S3 & 21.42 & 45.68 & 2.83 & 0.85 & 70.79 \\
\hline & S1 & 2.15 & 9.38 & 0.00 & 0.00 & 11.53 \\
\hline \multirow[t]{2}{*}{ Wheat } & S2 & 49.62 & 90.60 & 26.07 & 3.32 & 169.61 \\
\hline & S3 & 0.00 & 0.00 & 0.00 & 0.00 & 0.00 \\
\hline \multirow{3}{*}{ Maize } & S2 & 34.46 & 85.07 & 26.07 & 3.32 & 148.91 \\
\hline & S3 & 17.31 & 14.91 & 0.01 & 0.00 & 32.23 \\
\hline & $\mathrm{S} 1$ & 1.41 & 2.76 & 0.00 & 0.00 & 4.17 \\
\hline \multirow[t]{3}{*}{ Olives } & S2 & 36.99 & 96.46 & 20.04 & 2.77 & 156.27 \\
\hline & S3 & 13.36 & 0.76 & 6.03 & 0.54 & 20.70 \\
\hline & $\mathrm{S} 1$ & 0.74 & 6.62 & 0.00 & 0.00 & 7.36 \\
\hline \multirow[t]{2}{*}{ Cotton } & $\mathrm{S} 2$ & 22.25 & 55.72 & 26.06 & 3.32 & 107.35 \\
\hline & S3 & 28.78 & 37.64 & 0.01 & 0.00 & 66.43 \\
\hline
\end{tabular}


recommended as these applications evaluates the land based on the minimum limiting factor. LUSET application for land suitability or Modified Storie index for land capability is recommended where all soil parameters share together for assessing the soil suitability rate by calculating the average methods.

\section{REFERENCES}

Abd El-Aziz, S.H. (2018). Soil capability and suitability assessment of Tushka Area, Egypt by using different programs (ASLE, Microleis and Modified Storie Index). Malaysian Journal of Sustainable Agriculture. 2(2): 9-15.

Abd-Elmabod, S.K., Noura, B., Miriam, M., Paulo, P. Zhenhua, Z., Artemi, C., Antonio, J., Hani, M., Diego D., Laurence, J. (2019). Assessment of soil suitability for improvement of soil factors and agricultural management. Sustainability. 11(6): 1588 .

Aldabaa, A.A.A. (2018). Characterization of land suitability for crop and fruit production in Wadi Sakher at North West Coastal Zone of Egypt. Alexandria Science Exchange Journal. 39(4): 560-577.

Bhaskar, B.P., Bobade, S.V., Gaikwad, S.S., Sarkar, D., Anantwar, S.G., Bhattacharyya, T. (2015). Soil informatics for agricultural land suitability assessment in Seoni district, Madhya Pradesh, India. Indian J. Agric. Res. 49(4): 315-320.

De la Rosa, D., Anaya-Romero, M., Diaz-Pereira, E., Heredia, N., Shahbazi, F. (2009). Soil specific agro-ecological strategies for sustainable land use-A case study by using MicroLEIS DSS in Sevilla Province (Spain). Land Use Policy. 26(4): 1055-1065.

De la Rosa, D., Mayol, F., Fernandez, M. and Diaz-Pereira, E. (2004). A land evaluation decision support system (MicroLEIS DSS) for agricultural soil protection with special reference to the Mediterranean region. Environ Model Software. 19: 929-942.

Delgado F (2003). Soil Physical Properties on Venezu-elan Steeplands: Applications to Soil Conservation Planning, CIDIAT, University of Los Andes, Méri-da, Venezuela.

El Shazly, E., M. Abdel-Hady, M.El Ghawaby, I. El Kassas, S. Khawasik, M.El Shazly and S. Sanad. (1975). Geologic interpretation of Landsat satellite images for west Nile delta area, Egypt. Cairo, Egypt: The Remote Sensing Research Project, Academy of Scientific Research and Technology.

EMA (Egyptian Meteorological Authority) (2014). Climatic Atlas of Egypt. Ministry of Transport, Cairo, Egypt.

FAO (1976). A Framework for Land Evaluation. FAO Soils Bulletin vol. 32. Food and Agriculture Orga-nization, Rome.

FAO (2006). Guidelines for Soil Description, $4^{\text {th }}$ ed. Food and Agriculture Organization of the United Nations, Rome.

Ismail, H.A., M.H. Bahnassy, O.R. Abd El-Kawy, (2005). Integrating GIS and modelling for agricultural land suitability evaluation at East Wadi El-Natrun, Egypt. Egyptian Journal of Soil Science. 45: 297-322.
Klingebiel AA, Montgomery PH (1961). Land Capabi-lity Classification. USDA Handbook, 210, United States Department of Agriculture, Washington DC.

Mahmoud, E.A., Sayed, A.S.A., Aldabaa, A.A.A. (2019). Land capability classification of Wadi Jerafi Basin, North Sinai Egypt. Alexandria Science Exchange Journal. 40(1): 43-59.

Ramamurthy, V., Mamatha, D., Niranjan, K.V., Vasundhara, R., K., Ranjitha, Chandrakala, M., Singh, S.K. (2019). Suitability evaluation for pigeon pea in southern transition zone of Karnataka Plateau, India. Legume Research, DOI: 10.18805/LR-4047.

Sabareeshwari, V., Baskar, M., Shanmugam, P.M. (2018). Evaluation of soil site for suitability of maize and fertility mapping using GIS 10.1 in ponnaniyar basin, Trichy, Tamil Nadu, India. Agric. Sci. Digest. 38(2): 108-112.

Sawy, S., Abd El-Hady, A.A. and Yousif, I.A.H. (2013). Land evaluation and sustainable development of some Areas of Dakhla Oasis, Egypt. J.Soil Sci. and Agric. Eng., Mansoura Univ. 4(12): 1393-1409.

Singha, C., Swain, K.C. (2016). Land suitability evaluation criteria for agricultural crop selection: A review. Agricultural Reviews. 37(2): 125-132.

Soil Survey Staff. (2014). Key to Soil Taxonomy. Twelfth Edition, U.S.D.A., Washington, D.C. 372 p.

Storie RE (1973). An Index for Rating the Agricultural Valuve of Soils, University of California, Agricul-tural Experiment Station Berkley, California.

UCDAVIS, (2008). A Revised Storie Index for Use with Digital Soils Information. University of California Division of Agriculture and natural Resources. Publication No: 8335, 11p.

USA. Xingwu D, Li R, Guangli Z, Jinming H and Haiyan F (2015). Soil productivity in the Yunnan province: Spatial distribution and sustainable utilization. Soil and Tillage Research. 147: 10-19.

USDA (2017). Soil Survey Manual soil by Science Divison Staff. Agricuture Handbook No.18. USDA.

Yen B.T., Pheng, K.S. and Hoanh, C.T. (2006). Land Use Suitability Evaluation Tool (LUSET), GIS-IP Laboratory, International Rice Research Institute, Dapo, Box 7777, Metro Manila, Philippines, $222 \mathrm{p}$.

Yousif, I.A.H. (2018). Land Capability and Suitability Mapping in Some Areas of North-Western Coast, Egypt J. Soil Sci. and Agric. Eng., Mansoura Univ. 9(3): 111-118.

Yousif, I.A.H. (2019). Soil Suitability Assessment Using MicroLEIS Model: A Case Study in Wadi El Heriga, North Western Coast Zone, Egypt. Egypt. J. Soil Sci. 59(3): 209-221.

Yousif, I.A.H., Ahmed, A.S. (2019). Integration of land cover changes and land capability of Wadi El-Natrun depression using vegetation indices. Egyptian Journal of Soil Science. 59 (4): 385-402.

Zhang, J., Su, Y., Wu, J., Liang, H. (2015). GIS based land suitability assessment for tobacco production using AHP and fuzzy set in Shandong province of China. Comput. Electron. Agric. 114: 202-211. 